\title{
South Asian individuals with diabetes who are referred for MODY testing in the UK have a lower mutation pick-up rate than white European people
}

\author{
Shivani Misra ${ }^{1} \cdot$ Beverley Shields $^{2} \cdot$ Kevin Colclough ${ }^{3}$ - Desmond G Johnston ${ }^{1}$. \\ Nick S Oliver ${ }^{1}$ - Sian Ellard ${ }^{2,3}$ - Andrew T Hattersley ${ }^{2}$
}

Received: 10 May 2016 / Accepted: 24 June 2016 / Published online: 19 July 2016

(C) The Author(s) 2016. This article is published with open access at Springerlink.com

Keywords Glucokinase · Hepatic nuclear factor 1 alpha · Hepatic nuclear factor 4 alpha $\cdot$ MODY · South Asian

\section{Abbreviations \\ SA South Asian \\ WE White European}

To the Editor: Maturity onset diabetes of the young (MODY) is an autosomal dominantly inherited form of diabetes that has been extensively described in white populations, but diagnosed with lower frequency in other ethnic groups. Mutations in the $G C K, H N F 1 A$ and $H N F 4 A$ MODY genes have been reported in a variety of ethnic groups [1-3], but there are few data on prevalence in non-white ethnic groups. MODY was first reported in a UK South Asian (SA) cohort in a systematic survey of childhood diabetes [4]. A 2006 study revealed a lower than expected frequency of referrals for

Shivani Misra

s.misra@imperial.ac.uk

1 Diabetes, Endocrinology and Metabolism, Imperial College London, Ground Floor Medical School Building, St Mary's Campus, Norfolk Place, London W2 1PG, UK

2 Institute of Biomedical and Clinical Science, University of Exeter Medical School, Exeter, UK

3 Department of Molecular Genetics, Royal Devon and Exeter NHS Foundation Trust, Exeter, UK
MODY testing in the UK SA population [5]. The frequency and characteristics of MODY in non-white European populations is important for the precise diagnosis of young-onset diabetes.

We undertook a retrospective analysis of UK referrals for MODY testing to determine if referrals for individuals of SA origin had increased since that reported in the 2006 study and to investigate the pick-up rate and clinical features of MODY mutations in this population.

The UK MODY database, held centrally at the Royal Devon and Exeter National Health Service (NHS) Foundation Trust, is populated prospectively from a standardised request proforma that includes ethnicity. Proband referrals ( $>1$ year old at diagnosis) from 1996 to November 2015, in whom sequencing for mutations in the most common MODY genes (HNF1A, HNF4A, HNF1B or $G C K)$ was requested, were selected. Only variants classified as pathogenic or likely pathogenic were included. Assignment of pathogenicity was based on UK best practice guidelines [6]; including findings from established variant/mutation database and literature searches, details of familial cosegregation and identification of clinical features characteristic of the MODY gene mutation. Additionally, data from in silico prediction models (SIFT, PolyPhen2, AlignGVGD, Grantham distance accessed using AlaMut Visual 2.7, Interactive Biosoftware, Rouen, France) were used, along with details of amino acid species conservation and predictions of effects on splicing. Finally, variants in the Exome Aggregation Consortium [7] and 1000 genomes [8] databases with a minor allele frequency $>0.01$, were excluded.

Ethnicity was categorised as white European (WE) or SA based on UK census classification [9]. The SA group included people from India, Pakistan and Bangladesh or in whom ethnic origin was stated to be 'Asian' and excluded mixed ethnicity. 
A total of 4688 proband referrals were identified and ethnicity was recorded in 4010 cases. White European referrals accounted for 3472 of 4010 (86.6\%). 293 cases referred were in people of SA ethnicity $(7.3 \%)$, and 81 referrals $(2.0 \%)$ were from African or Caribbean backgrounds (see Table 1).

1180 probands had MODY mutations, with a mutation pick-up rate of $25.2 \%(1180 / 4688)$. The mutation pick-up rate was $29.1 \%(1011 / 3472)$ in the WE group and $12.6 \%(37 / 293)$ in the SA group $(p<0.001)$ (see Table 1$)$. The pick-up rate in children $(<12$ years) referred for testing did not differ between ethnic groups; $32.6 \%$ in WE vs $26.7 \%$ in SA children, $p=0.356$ (data not shown).

Phenotypes of those with and without confirmed MODY mutations are displayed in Table 2. Generally, SA MODY patients with $G C K$ mutations were diagnosed earlier than WE patients (6.5 vs 20 years, $p<0.0001)$; however, when only considering diagnosis in children under 12 years old, there was no significant difference in age at diagnosis. Referrals from both ethnic groups had similar proportions of a history of parental diabetes and were equally likely to be non-insulin dependent.

In both SA and WE individuals, the HNF1A / HNF4A MODY phenotypes were similar across all variables reported except for $\mathrm{HbA}_{1 \mathrm{c}}$, which was higher in the $\mathrm{SA}$ group $(8.6 \%$ [70 $\mathrm{mmol} / \mathrm{mol}]$ vs $7.2 \%$ [55 $\mathrm{mmol} / \mathrm{mol}], p=0.004$; see Table 2).

SA people in whom no MODY mutations were found were younger at diagnosis (19 years vs 25 years, $p<0.0001)$ and had a shorter duration of diabetes, compared with WE individuals. BMI was similar across both ethnic groups (WE, 25.3 vs SA $25.0 \mathrm{~kg} / \mathrm{m}^{2}, p=0.217$ ). However, SA people were more likely to have a parent affected with diabetes $(83.6 \%$ vs $68.2 \%, p<0.0001)$ and were more likely not to be treated with insulin at referral $(60.2 \%$ vs $50.6 \%, p=0.006)$, when compared with WE individuals (see Table 2).

This study is the first to explore the characteristics of individuals of SA ethnicity who have been referred for MODY testing using data from the largest MODY diagnostic service in the UK. It demonstrates a lower pick-up rate of MODY mutations in people of SA ethnicity. In our analysis, SA people accounted for markedly more referrals than previously reported in 2006 ( $7.3 \%$ vs $0.5 \%)$ [5]. Whilst this broadly reflects the UK population demographics, because of the higher prevalence of type 2 diabetes in SA people it is suggested that this is still lower than expected [9]. The detection rate for MODY mutations was lower in the SA group than in WE referrals. However, when a mutation was not detected, SA referrals were more likely to meet the clinical criteria for MODY than WE individuals. This may demonstrate a referral bias favouring SA cases that more stringently meet the clinical criteria. However, the results also show that these criteria are not sensitive for the separation of MODY from young-onset type 2 diabetes in this population. For example, SA people without mutations had higher BMIs than those with confirmed MODY mutations, suggesting that the low MODY detection rate in this group may have resulted from a higher prevalence of young-onset, familial diabetes.

When analysing the SA group as a whole, the best predictors differentiating those with MODY (mutations in all genes) from those without a mutation, were a lower BMI and age at onset; BMI was lower in the SA group with MODY mutations than those without mutations (MODY, $21.4 \mathrm{~kg} / \mathrm{m}^{2}$ and no MODY, $25.0 \mathrm{~kg} / \mathrm{m}^{2}, p=0.001$, data not shown), but BMI was generally higher and non-significantly different in the no mutation group when compared with WE people (25.0 vs $25.3 \mathrm{~kg} / \mathrm{m}^{2}$, respectively). The lower BMI and age at diagnosis in SA referrals was primarily observed in the $G C K$ mutation group and not seen in the HNF1A / HNF4A mutation or 'no mutation' groups. It is likely that these findings reflect a lower referral rate in adults and children over 12 years old since the mutation pick-up rate in both ethnic groups was similar in children under 12 years.

There is limited evidence to support that SA individuals have a lower prevalence of MODY, with only one previous study suggesting fewer $G C K$ mutations
Table 1 Breakdown of proband referrals for MODY genetic testing and MODY pick-up rate by ethnic group

\begin{tabular}{lllll}
\hline Ethnic group & $n$ & $\%$ & MODY mutation $(n)$ & Pick-up rate (\%) \\
\hline All groups & 4688 & & 1180 & 25.2 \\
Individuals with known ethnicity & 4010 & & 1083 & 27.0 \\
$\quad$ White & 3472 & 86.6 & 1011 & 29.1 \\
South Asian & 293 & 7.3 & 37 & $12.6^{* * *}$ \\
African-Caribbean & 81 & 2 & 8 & 9.9 \\
Middle Eastern & 47 & 1.2 & 5 & 10.6 \\
South East Asian & 21 & 0.5 & 6 & 28.6 \\
Mixed/other & 96 & 2.4 & 16 & 16.7 \\
All non-white ethnic groups & 538 & 13.4 & 72 & 13.4 \\
\hline
\end{tabular}

Statistical analysis was carried out using a $\chi^{2}$ test on the two largest ethnic groups, white and South Asian *** $p<0.001$ vs white individuals 


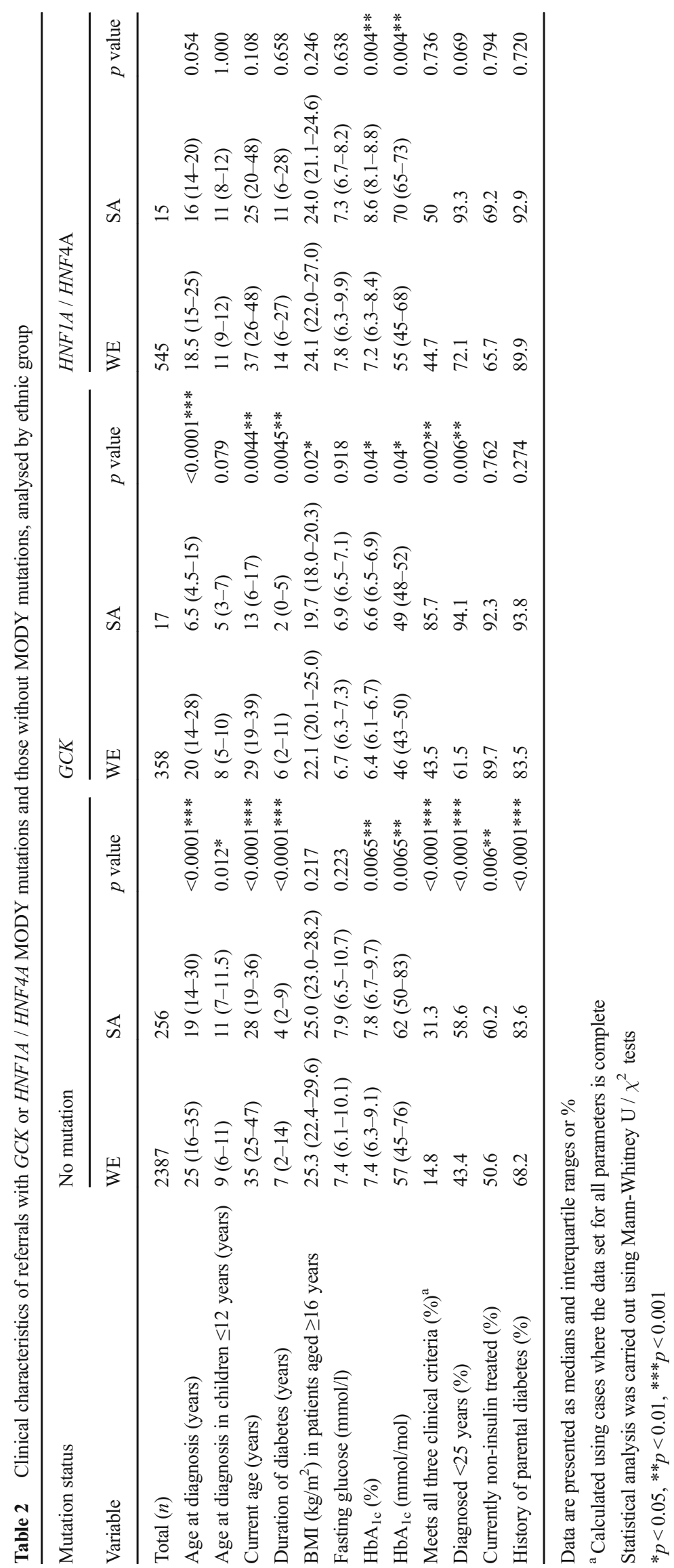


occurred in Indian children [10]; however, this study may have been underpowered since the estimated minimum prevalence of MODY (in a predominantly white population) has recently been demonstrated to be 1 in 1000 cases [11]. Thus, it is more likely that the lower mutation pick-up rate in SA individuals compared to WE individuals observed in our study reflects higher prevalence of younger-onset type 2 diabetes, which is supported by our analysis demonstrating similar mutation pick-up rates between ethnic groups in pre-pubertal children.

Our data highlight the need to consider the optimum method for identifying cases of MODY in people of non-white European ethnicity. Approaches such as the MODY probability calculator [12] need to be validated in non-white populations as our data suggest that MODY cases may be more sensitively identified using lower cut-offs for age at diagnosis and BMI.

Referrals for MODY testing in the SA population have increased considerably but may still be under-representative of the general UK population. Meanwhile, despite meeting referral criteria more stringently, the lower MODY mutation pick-up rate for SA individuals suggests that these criteria are not sensitive for separating MODY from younger-onset type 2 diabetes in the UK SA population.

Further work is required in unselected cohorts, to develop ethnic-specific criteria that facilitate the identification of MODY from type 2 diabetes in SA people with young-onset diabetes.

Funding SM is funded by the Diabetes, Research and Wellness Foundation Sutherland Earl Clinical Research Fellowship. BS, KC, SE, and ATH are supported by the National Institute of Health Research (NIHR)-funded Exeter Clinical Research Facility. SE and ATH are Wellcome Trust Senior Investigators.

Duality of interest The authors declare that there is no duality of interest associated with this manuscript.

Contribution statement SM and ATH designed the study. SM collated the data, carried out the analysis and drafted the manuscript. BS assisted with study design and provided support with analysis. $\mathrm{KC}$ assisted with data acquisition. DJ, NO, SE and ATH provided supervision, assisted with interpretation of analysis and revised the manuscript. All authors critically reviewed and revised the manuscript and approved the final version. ATH is the guarantor of this work.
Open Access This article is distributed under the terms of the Creative Commons Attribution 4.0 International License (http:// creativecommons.org/licenses/by/4.0/), which permits unrestricted use, distribution, and reproduction in any medium, provided you give appropriate credit to the original author(s) and the source, provide a link to the Creative Commons license, and indicate if changes were made.

\section{References}

1. Pihoker C, Gilliam LK, Ellard S et al (2013) Prevalence, characteristics and clinical diagnosis of maturity onset diabetes of the young due to mutations in HNF1A, HNF4A, and glucokinase: results from the SEARCH for diabetes in youth. J Clin Endocrinol Metab 98:4055-4062

2. Yorifuji T, Fujimaru R, Hosokawa Y et al (2012) Comprehensive molecular analysis of Japanese patients with pediatric-onset MODY-type diabetes mellitus. Pediatr Diabetes 13:26-32

3. Chapla A, Mruthyunjaya MD, Asha HS et al (2015) Maturity onset diabetes of the young in India- a distinctive mutation pattern identified through targeted next-generation sequencing. Clin Endocrinol (Oxf) 82:533-542

4. Ehtisham S, Hattersley AT, Dunger DB, Barrett TG (2004) First UK survey of paediatric type 2 diabetes and MODY. Arch Dis Child 89:526-529

5. Porter JR, Rangasami JJ, Ellard S et al (2006) Asian MODY: are we missing an important diagnosis? Diabet Med 23:1257-1260

6. Wallis Y, Payne S, McAnulty C et al (2013) Practice guidelines for the evaluation of pathogenicity and the reporting of sequence variants in clinical molecular genetics. http://www.ukcgg. org/media/774853/evaluation_and_reporting_of_sequence variants_bpgs_june_2013__finalpdf.pdf, accessed 19 June 2016

7. ExAC Exome Aggregation Consortium. http://exac.broadinstitute.org/, accessed 19 June 2016

8. 1000 genomes database. http://www1000genomes.org/, accessed 19 June 2016

9. http://www.ons.gov.uk/peoplepopulationandcommunity/culturalid entity/ethnicity/articles/ethnicityandnationalidentityinenglandandw ales/2012-12-11 UK Census Ethnicity Data, accessed 30 June 2016

10. Kanthimathi S, Jahnavi S, Balamurugan K et al (2014) Glucokinase gene mutations (MODY 2) in Asian Indians. Diabetes Technol Ther 16:180-185

11. Chakera AJ, Spyer G, Vincent N et al (2014) The $0.1 \%$ of the population with glucokinase monogenic diabetes can be recognized by clinical characteristics in pregnancy: the Atlantic Diabetes in Pregnancy cohort. Diabetes Care 37:1230-1236

12. Shields BM, McDonald TJ, Ellard S et al (2012) The development and validation of a clinical prediction model to determine the probability of MODY in patients with young-onset diabetes. Diabetologia 55:1265-1272 